https://helda.helsinki.fi

Quasiminimal structures and excellence

Bays, Martin

2014-02

Bays , M , Hart , B , Hyttinen , T , Kesälä , M \& Kirby , J 2014 , ' Quasiminimal structures and excellence ' , Bulletin of the London Mathematical Society , vol. 46 , pp. 155-163 . https://doi.org/10.1112/blms/bdt07

http://hdl.handle.net/10138/43175

https://doi.org/10.1112/blms/bdt076

publishedVersion

Downloaded from Helda, University of Helsinki institutional repository.

This is an electronic reprint of the original article.

This reprint may differ from the original in pagination and typographic detail.

Please cite the original version. 


\title{
Quasiminimal structures and excellence
}

\author{
Martin Bays, Bradd Hart, Tapani Hyttinen, Meeri Kesälä and Jonathan Kirby
}

\begin{abstract}
We show that the excellence axiom in the definition of Zilber's quasiminimal excellent classes is redundant, in that it follows from the other axioms. This substantially simplifies a number of categoricity proofs.
\end{abstract}

\section{Introduction}

The notion of a quasiminimal excellent class was introduced by Zilber [12] in order to prove categoricity of his non-elementary theories of covers of the multiplicative group of a field (group covers) [13] and of pseudoexponential fields [11]. The excellence axiom is the most technical part, and is adapted from Shelah's work on excellent sentences of $L_{\omega_{1}, \omega}$ (see [8]). Both Shelah's and Zilber's work on excellence is described in Baldwin's monograph [1]. Zilber's original proof of categoricity of group covers contained a gap, which was corrected in [3] by strengthening a hypothesis in one of the statements relating to excellence and giving a new proof. However, the proof of the categoricity of pseudoexponential fields relied on the original stronger and now unproved statement from [13]. A patch for the categoricity proof for pseudoexponential fields was recently circulated by the first and fifth authors [2].

In this paper, we show that the excellence axiom of quasiminimal excellence classes is actually redundant, in that it follows from the other axioms. This substantially simplifies the proof of categoricity of Zilber's group covers and pseudoexponential fields, and avoids the troublesome part of the proofs where the gaps were.

In the case of first-order theories, part of Shelah's Main Gap theorem involves reducing a condition on $n$-systems of models, akin to excellence, to the case $n=2$, where it becomes the condition (prime models over pairs) that primary models exist over independent pairs of models $[\mathbf{4}, \mathbf{9}]$. The main insight behind the current paper is that these arguments, suitably modified, apply also to the (non-elementary) classes of structures considered here, and moreover that the reduction can be pushed even further, to $n=1$, where the condition becomes one of $\aleph_{0}$-stability over models. This reduction is performed in Proposition 6.2. In Propositions 4.2 and 5.2 , we find that this $\aleph_{0}$-stability condition does follow from the $\aleph_{0}$-homogeneity over models assumed of quasiminimal excellent classes. This argument is based on a classical argument from stability theory, but the version in this paper is a modification of a corresponding argument in the non-elementary framework of finitary abstract elementary classes [5].

An uncountable structure $M$ is quasiminimal if every first-order $M$-definable subset of $M$ is countable or co-countable. In Section 7, we consider in the light of our main results the question of when a quasiminimal structure belongs to a quasiminimal excellent class.

Our main results directly answer Question 1 in [6, Section 6]. They also render Question 2 there redundant: it asks for equivalence of the excellence axiom and the conclusion of $[\mathbf{6}$, Lemma 3.2], which we show both to be consequences of the other axioms, hence trivially

Received 21 October 2012; revised 3 May 2013; published online 24 October 2013.

2010 Mathematics Subject Classification 03C45 (primary).

The fourth author was funded by the Academy of Finland, project number 1251557. 
equivalent modulo them. The remaining questions, $3-5$, concern finite-dimensional models; our techniques say little about these, and in fact it is key to the proof of our main result that we deal only with infinite-dimensional models.

\section{Statement of main result}

Throughout this paper, the notion of type will be quantifier-free $L$-type, denoted by tp. It will follow from our axioms that if finite tuples $\bar{a}$ and $\bar{b}$ from a model satisfy the same quantifier-free $L$-type, then they satisfy the same complete type (and even the same $L_{\infty, \omega}$-type), justifying our notation. In applications, this is usually achieved by expanding the language. However, it does not necessarily follow that the first-order theory of our models has quantifierelimination, since not all types of the first-order theory are necessarily realized in the models we consider.

Definition 2.1. Let $M$ be an $L$-structure for a countable language $L$, equipped with a pregeometry $\mathrm{cl}$ (or $\mathrm{cl}_{M}$ if it is necessary to specify $M$ ). We say that $M$ is a quasiminimal pregeometry structure if the following hold.

(QM1) The pregeometry is determined by the language. That is, if $\operatorname{tp}(a, \bar{b})=\operatorname{tp}\left(a^{\prime}, \bar{b}^{\prime}\right)$, then $a \in \operatorname{cl}(\bar{b})$ if and only if $a^{\prime} \in \operatorname{cl}\left(\bar{b}^{\prime}\right)$.

(QM2) The structure $M$ is infinite-dimensional with respect to $\mathrm{cl}$.

(QM3) (Countable closure property). If $A \subseteq M$ is finite, then $\operatorname{cl}(A)$ is countable.

(QM4) (Uniqueness of the generic type). Suppose that $H, H^{\prime} \subseteq M$ are countable closed subsets, enumerated such that $\operatorname{tp}(H)=\operatorname{tp}\left(H^{\prime}\right)$. If $a \in M \backslash H$ and $a^{\prime} \in M \backslash H^{\prime}$, then $\operatorname{tp}(H, a)=$ $\operatorname{tp}\left(H^{\prime}, a^{\prime}\right)$ (with respect to the same enumerations for $H$ and $H^{\prime}$ ).

(QM5) ( $\aleph_{0}$-homogeneity over closed sets and the empty set). Let $H, H^{\prime} \subseteq M$ be countable closed subsets or empty, enumerated such that $\operatorname{tp}(H)=\operatorname{tp}\left(H^{\prime}\right)$, and let $\bar{b}, \bar{b}^{\prime}$ be finite tuples from $M$ such that $\operatorname{tp}(H, \bar{b})=\operatorname{tp}\left(H^{\prime}, \bar{b}^{\prime}\right)$, and let $a \in \operatorname{cl}(H, \bar{b})$. Then there is $a^{\prime} \in M$ such that $\operatorname{tp}(H, \bar{b}, a)=\operatorname{tp}\left(H^{\prime}, \bar{b}^{\prime}, a^{\prime}\right)$.

We say $M$ is a weakly quasiminimal pregeometry structure if it satisfies all the axioms except possibly (QM2).

Note that, while in (QM5) there is a restriction that $a \in \operatorname{cl}(H \bar{b})$, in the presence of the other axioms this restriction can be removed. In particular, we have the usual notion of $\aleph_{0^{-}}$ homogeneity of a structure.

Lemma 2.2. Let $M$ be a weakly quasiminimal pregeometry structure, let $\bar{b}, \bar{b}^{\prime}$ be finite tuples from $M$ such that $\operatorname{tp}(\bar{b})=\operatorname{tp}\left(\bar{b}^{\prime}\right)$, and let $\bar{a}$ be a finite tuple from $M$. Then there is $\bar{a}^{\prime}$ in $M$ such that $\operatorname{tp}(\bar{a} \bar{b})=\operatorname{tp}\left(\bar{a}^{\prime} \bar{b}^{\prime}\right)$.

Proof. We may assume that $\bar{a}$ is a singleton, $a$. If $a \in \operatorname{cl}(\bar{b})$, then apply (QM5). If not, since cl is a pregeometry and using (QM1) we have $\operatorname{dim} M \geqslant \operatorname{dim}(a \bar{b})=\operatorname{dim}(\bar{b})+1=\operatorname{dim}\left(\bar{b}^{\prime}\right)+1$. So there is $c \in M \backslash \operatorname{cl}\left(\bar{b}^{\prime}\right)$, and by (QM4) we can take $a^{\prime}$ to be any such $c$.

Given $M_{1}$ and $M_{2}$ both weakly quasiminimal pregeometry $L$-structures, we say that an $L$-embedding $\theta: M_{1} \hookrightarrow M_{2}$ is a closed embedding if for each $A \subseteq M_{1}$ we have $\theta\left(\operatorname{cl}_{M_{1}}(A)\right)=$ $\mathrm{cl}_{M_{2}}(\theta(A))$. In particular, $\theta\left(M_{1}\right)$ is closed in $M_{2}$ with respect to $\mathrm{cl}_{M_{2}}$. We write $M_{1} \preccurlyeq$ cl $M_{2}$ for a closed embedding. 
Given a quasiminimal pregeometry structure $M$, let $\mathcal{K}^{-}(M)$ be the smallest class of $L$-structures that contains $M$ and all its closed substructures and is closed under isomorphism, and let $\mathcal{K}(M)$ be the smallest class containing $\mathcal{K}^{-}(M)$ that is also closed under taking unions of chains of closed embeddings. Then both $\mathcal{K}^{-}(M)$ and $\mathcal{K}(M)$ satisfy axioms 0 , I, and II of quasiminimal excellent classes from $[6]$, and $\mathcal{K}(M)$ also satisfies axiom IV and, together with closed embeddings, forms an abstract elementary class. We call any class of the form $\mathcal{K}(M)$ a quasiminimal class.

Our main result is the following theorem.

Theorem 2.3. If $\mathcal{K}$ is a quasiminimal class, then every structure $A \in \mathcal{K}$ is a weakly quasiminimal pregeometry structure, and up to isomorphism there is exactly one structure in $\mathcal{K}$ of each cardinal dimension. In particular, $\mathcal{K}$ is uncountably categorical. Furthermore, $\mathcal{K}$ is the class of models of an $L_{\omega_{1}, \omega}(Q)$ sentence.

When $M$ satisfies an additional property called excellence, Theorem 2.3 is Zilber's main categoricity theorem, specifically in the form from [6, Theorem 4.2 and Corollary 5.7], along with the $L_{\omega_{1}, \omega}(Q)$-definability result [6, Theorem 5.5]. We will prove Theorem 2.3 by showing in Proposition 6.2 that the specific form of the excellence property used in the categoricity proof always holds.

Assuming that proposition, we prove the main theorem.

Proof of Theorem 2.3. Let $\mathfrak{M}$ be a quasiminimal pregeometry structure and $\mathcal{K}=\mathcal{K}(\mathfrak{M})$. As in [6, Theorem 2.2], all closed subsets of $\mathfrak{M}$ of dimension $\aleph_{0}$ are isomorphic to each other, and are also quasiminimal pregeometry structures. Let $M$ be one. Then, by Proposition 6.2, $M$ satisfies the excellence property. Thus, by Kirby [6, Corollary 5.7 and Theorem 4.2], $\mathcal{K}(M)$ is a quasiminimal excellent class and has exactly one model of each cardinal dimension, and by Kirby [6, Theorem 5.5] it is the class of models of an $L_{\omega_{1}, \omega}(Q)$ sentence. It remains to show that $\mathcal{K}=\mathcal{K}(M)$. Let $B$ be a basis for $\mathfrak{M}$, and note that $\mathfrak{M}=\bigcup\left\{\operatorname{cl}\left(B^{\prime}\right)\left|B^{\prime} \subseteq B,\right| B^{\prime} \mid=\aleph_{0}\right\}$. Since $\mathcal{K}(M)$ is closed under unions of chains, it is also closed under unions of directed systems, and hence $\mathfrak{M} \in \mathcal{K}(M)$. Thus, $\mathcal{K}(\mathfrak{M})=\mathcal{K}(M)$.

\section{Models and types}

Let $\mathcal{K}$ be a quasiminimal class. We call the structures in $\mathcal{K}$ models. Then, by Kirby [6, Theorem 2.1], the models of dimension up to $\aleph_{1}$ are determined up to isomorphism by their dimension. Furthermore, back-and-forth arguments as in the proof of that theorem immediately give us the following characterization of types.

Lemma 3.1. Let $\mathfrak{M}$ be a model of dimension at most $\aleph_{1}$, let $M \preccurlyeq c \mathrm{cl}$ with $M$ countable, let $H=\emptyset$ or $H \preccurlyeq_{\mathrm{cl}} M$, and let $\bar{a}, \bar{b}$ be $n$-tuples from $M$. Then the following are equivalent:

(i) $\operatorname{tp}(\bar{a} / H)=\operatorname{tp}(\bar{b} / H)$;

(ii) there exists $f \in \operatorname{Aut}(M / H)$ with $f(\bar{a})=\bar{b}$;

(iii) there exists $f \in \operatorname{Aut}(\mathfrak{M} / H)$ with $f(\bar{a})=\bar{b}$;

(iv) there exists $f \in \operatorname{Aut}(\mathfrak{M} / H)$ with $f(\bar{a})=\bar{b}$ and $f(M)=M$.

Thus, Galois types coincide with syntactic types for types over the empty set and over models, and furthermore Galois types do not depend on the model in which they are calculated (we have shown this for models of dimension at most $\aleph_{1}$, but it will follow from our main result that it holds for arbitrary models). 


\section{Splitting of types}

Definition 4.1. Let $\mathfrak{M}$ be a model, and let $B \subseteq \mathfrak{M}$ and $\bar{a} \in \mathfrak{M}$. We say that $\operatorname{tp}(\bar{a} / B)$ splits over a finite $A \subseteq B$ if there are finite tuples $\bar{c}$ and $\bar{d}$ in $B$ with

$$
\begin{aligned}
& \operatorname{tp}(\bar{c} / A)=\operatorname{tp}(\bar{d} / A) \quad \text { but } \\
& \operatorname{tp}(\bar{c} / A \cup \bar{a}) \neq \operatorname{tp}(\bar{d} / A \cup \bar{a}) .
\end{aligned}
$$

Proposition 4.2. Let $\mathfrak{M}$ be a model and $M \preccurlyeq c l ~ \mathfrak{M}$ be a countable closed submodel. For each finite tuple $\bar{a} \in \mathfrak{M}$, there is a finite $A \subseteq M$ such that $\operatorname{tp}(\bar{a} / M)$ does not split over $A$.

Proof. Replacing $\mathfrak{M}$ with $\operatorname{cl}(M \bar{a})$, we may assume $\mathfrak{M}$ to be countable. If $M$ has finite cardinality, then we may take $A=M$. So assume $|M|=\aleph_{0}$.

We suppose that no such finite $A$ exists and construct uncountably many types over $M$, all realized in $\mathfrak{M}$. This contradicts the countability of $\mathfrak{M}$.

Enumerate $M=\left\{e_{n}: n<\omega\right\}$. For each $k<\omega$ and $\eta: k \rightarrow 2$, we denote by $\eta \frown 0$ and $\eta \frown 1$ the functions with domain $k+1$ extending $\eta$ and mapping $k$ to 0 and 1 , respectively. Given any function $f$ and a subset $A$ of the domain of $f$, we write $f \mid A$ for the restricted function.

We recursively construct finite sets $A_{\eta}$ and automorphisms $\sigma_{\eta} \in \operatorname{Aut}(\mathfrak{M})$ such that:

(i) $\sigma_{\eta}(M)=M$;

(ii) $\eta \subset \tau$ implies $\sigma_{\tau}\left|A_{\eta}=\sigma_{\eta}\right| A_{\eta}$;

(iii) for any $\mu: \omega \rightarrow 2$, we have that

$$
M=\bigcup_{k<\omega} A_{\mu \mid k}
$$

and that

$$
M=\bigcup_{k<\omega} \sigma_{\mu \mid k}\left(A_{\mu \mid k}\right)
$$

(iv) $\operatorname{tp}\left(\sigma_{\eta \frown 0}(\bar{a}) / B_{\eta}\right) \neq \operatorname{tp}\left(\sigma_{\eta-1}(\bar{a}) / B_{\eta}\right)$ where

$$
B_{\eta}=\sigma_{\eta \frown 0}\left(A_{\eta \frown 0}\right) \cap \sigma_{\eta \frown 1}\left(A_{\eta \frown 1}\right) \subset M .
$$

First let $A_{\emptyset}=\emptyset$ and $\sigma_{\emptyset}=\operatorname{Id}_{\mathfrak{M}}$. Then assume that we have defined these for all $\eta$ with domain $\leqslant k$.

Since $\operatorname{tp}(\bar{a} / M)$ splits over $A_{\eta}$ by assumption, there are finite $\bar{c}, \bar{d} \in M$ with

$$
\begin{aligned}
& \operatorname{tp}\left(\bar{c} / A_{\eta}\right)=\operatorname{tp}\left(\bar{d} / A_{\eta}\right) \quad \text { but } \\
& \operatorname{tp}\left(\bar{c} / A_{\eta} \cup \bar{a}\right) \neq \operatorname{tp}\left(\bar{d} / A_{\eta} \cup \bar{a}\right) .
\end{aligned}
$$

Hence, there is $f \in \operatorname{Aut}\left(\mathfrak{M} / A_{\eta}\right)$ mapping $\bar{c}$ to $\bar{d}$ and by Lemma 3.1 we may assume that $f(M)=M$.

Let $\sigma_{\eta \frown 0}=\sigma_{\eta}$ and $\sigma_{\eta-1}=\sigma_{\eta} \circ f$. Furthermore, for $i=0,1$ let

$$
A_{\eta \frown i}=A_{\eta} \cup\left\{e_{k+1}, \sigma_{\eta \frown}^{-1}\left(e_{k+1}\right), \bar{d}, \bar{c}\right\} .
$$

We have that

$$
\sigma_{\eta \frown 1}\left|A_{\eta}=\sigma_{\eta \frown 0}\right| A_{\eta}=\sigma_{\eta} \mid A_{\eta},
$$

and that

$$
\sigma_{\eta-1}(\bar{c})=\sigma_{\eta}(\bar{d})=\sigma_{\eta \frown 0}(\bar{d}) .
$$

Hence, $\sigma_{\eta}\left(A_{\eta}\right)$ and $\sigma_{\eta}(\bar{d})$ are in the set $B_{\eta}$ of item (iv). 
Now item (iv) must hold, since if there were $g \in \operatorname{Aut}\left(\mathfrak{M} / \sigma_{\eta}\left(A_{\eta}\right) \cup \sigma_{\eta}(\bar{d})\right)$ mapping $\sigma_{\eta}-_{0}(\bar{a})$ to $\sigma_{\eta-1}(\bar{a})$, then the automorphism $\sigma_{\eta-1}^{-1} \circ g \circ \sigma_{\eta-0}$ would map $\bar{d}$ to $\bar{c}$ and fix $\bar{a}$ and $A_{\eta}$, contradicting splitting.

Finally, we define for each $\mu: \omega \rightarrow 2$ a map $f_{\mu}$ as the union of the restricted maps $\sigma_{\mu \mid k}$ on $A_{\mu \mid k}$ for $k<\omega$. By item (ii), the map is well defined and by item (iii) it is an automorphism of $M$. By Lemma 3.1, each $f_{\mu}$ extends to an automorphism $\pi_{\mu}$ of $\mathfrak{M}$.

Now suppose that $\mu, \nu: \omega \rightarrow 2$ are distinct, let $k$ be greatest such that $\mu|k=\nu| k$, and let $\eta=\mu \mid k$. Then without loss of generality, $\mu \mid k+1=\eta \frown 0$ and $\nu \mid k+1=\eta \frown 1$. Thus, $\pi_{\mu} \mid A_{\eta \frown 0}=$ $\sigma_{\eta \frown 0} \mid A_{\eta \frown 0}$, so

$$
\operatorname{tp}\left(\pi_{\mu}(\bar{a}) / \sigma_{\eta \frown 0}\left(A_{\eta}-0\right)\right)=\operatorname{tp}\left(\pi_{\mu}(\bar{a}) / \pi_{\mu}\left(A_{\eta \frown 0}\right)\right)=\operatorname{tp}\left(\sigma_{\eta \frown 0}(\bar{a}) / \sigma_{\eta \frown 0}\left(A_{\eta \frown 0}\right)\right) .
$$

Since $B_{\eta} \subseteq \sigma_{\eta \frown 0}\left(A_{\eta} \frown 0\right)$, we have $\operatorname{tp}\left(\pi_{\mu}(\bar{a}) / B_{\eta}\right)=\operatorname{tp}\left(\sigma_{\eta \frown 0}(\bar{a}) / B_{\eta}\right)$.

The same argument shows that $\operatorname{tp}\left(\pi_{\nu}(\bar{a}) / B_{\eta}\right)=\operatorname{tp}\left(\sigma_{\eta}-1(\bar{a}) / B_{\eta}\right)$.

Thus, by item (iv), $\operatorname{tp}\left(\pi_{\mu}(\bar{a}) / B_{\eta}\right) \neq \operatorname{tp}\left(\pi_{\nu}(\bar{a}) / B_{\eta}\right)$ and hence $\operatorname{tp}\left(\pi_{\mu}(\bar{a}) / M\right) \neq \operatorname{tp}\left(\pi_{\nu}(\bar{a}) / M\right)$. Thus, we have $2^{\aleph_{0}}$ different types over $M$, all realized in $\mathfrak{M}$, which is a contradiction.

\section{Isolation of types}

Definition 5.1. Let $A$ be a subset of a model $\mathfrak{M}$ and let $\bar{a} \in \operatorname{cl}(A)$. We say that the $\operatorname{tp}(\bar{a} / A)$ is s-isolated if there is a finite subset $A_{0} \subseteq A$ such that if $\bar{b} \in \operatorname{cl}(A)$ and $\operatorname{tp}\left(\bar{b} / A_{0}\right)=\operatorname{tp}\left(\bar{a} / A_{0}\right)$, then $\operatorname{tp}(\bar{b} / A)=\operatorname{tp}(\bar{a} / A)$. In this case, we also say $\operatorname{tp}(\bar{a} / A)$ is s-isolated over $A_{0}$.

In Shelah's notation, this is $F_{\aleph_{0}}^{s}$-isolation [9, p. 157]. In general, it does not imply isolation of a type by a single formula, at least not without expanding the language.

We show that types of tuples inside the closure of a model union a finite set are s-isolated.

Proposition 5.2. Let $\mathfrak{M}$ be a model, and let $M \preccurlyeq$ cl $\mathfrak{M}$ be a countable closed submodel. Let $\bar{a}, \bar{b} \in \mathfrak{M}$ be finite tuples with $\bar{b} \in \operatorname{cl}(M \bar{a})$. Then $\operatorname{tp}(\bar{b} / M \cup \bar{a})$ is s-isolated.

To show that the hypotheses cannot be significantly weakened, consider a quasiminimal pregeometry structure $\mathfrak{M}$ where the language contains a single equivalence relation, and $\mathfrak{M}$ has $\aleph_{0}$ equivalence classes, all of size $\aleph_{0}$. For $A \subseteq \mathfrak{M}, \operatorname{cl}(A)$ is the union of the equivalence classes that meet $A$. Then if $M \subseteq \mathfrak{M}$ is infinite but not closed, then the conclusion fails.

Proof of Proposition 5.2. By Proposition 4.2, there exists a finite $A \subset M$ such that $\operatorname{tp}(\bar{a} \bar{b} / M)$ does not split over $A$. We may suppose (extending $A$ ) that $\bar{a}$ is cl-independent from $M$ over $A$, and that $\bar{b} \in \operatorname{cl}(A \bar{a})$. We will show that $\operatorname{tp}(\bar{b} / M \bar{a})$ is s-isolated over $A \bar{a}$.

Let $\bar{b}^{\prime} \in \mathfrak{M}$ with $\operatorname{tp}\left(\bar{b}^{\prime} / A \bar{a}\right)=\operatorname{tp}(\bar{b} / A \bar{a})$. Let $\bar{d} \in M$.

Claim 1. There exists $\bar{d}^{\prime} \in M$ such that $\operatorname{tp}\left(\bar{b}^{\prime} \bar{d} / A \bar{a}\right)=\operatorname{tp}\left(\bar{b} \bar{d}^{\prime} / A \bar{a}\right)$.

Assume the claim. Then $\operatorname{tp}(\bar{d} / A)=\operatorname{tp}\left(\bar{d}^{\prime} / A\right)$, so by non-splitting $\operatorname{tp}(\bar{d} / A \bar{a} \bar{b})=\operatorname{tp}\left(\bar{d}^{\prime} / A \bar{a} \bar{b}\right)$. Hence, $\quad \operatorname{tp}(\bar{b} \bar{d} / A \bar{a})=\operatorname{tp}\left(\bar{b} \bar{d}^{\prime} / A \bar{a}\right)=\operatorname{tp}\left(\bar{b}^{\prime} \bar{d} / A \bar{a}\right), \quad$ and $\quad$ so $\quad \operatorname{tp}(\bar{b} / A \bar{d} \bar{a})=\operatorname{tp}\left(\bar{b}^{\prime} / A \bar{d} \bar{a}\right)$. So $\operatorname{tp}(\bar{b} / M \bar{a})=\operatorname{tp}\left(\bar{b}^{\prime} / M \bar{a}\right)$.

It remains to prove the claim. Say $\bar{d}=\bar{d}_{1} \bar{d}_{2}$ with $\bar{d}_{1}$ an independent tuple over $\operatorname{cl}(A \bar{a})$ and $\bar{d}_{2} \in \operatorname{cl}\left(A \bar{a} \bar{d}_{1}\right)$. By the independence of $\bar{a}$ from $M$ over $A$, in fact $\bar{d}_{2} \in \operatorname{cl}\left(A \bar{d}_{1}\right)$. Since $\bar{b} \in \operatorname{cl}(A \bar{a})$, by $(\mathrm{QM} 4)$ we have $\operatorname{tp}\left(\bar{b}^{\prime} / A \bar{a} \bar{d}_{1}\right)=\operatorname{tp}\left(\bar{b} / A \bar{a} \bar{d}_{1}\right)$. So, by Lemma 2.2 , there exists $\bar{d}_{2}^{\prime} \in \mathfrak{M}$ such that $\operatorname{tp}\left(\bar{b}^{\prime} \bar{d}_{2} / A \bar{a} \bar{d}_{1}\right)=\operatorname{tp}\left(\bar{b} \bar{d}_{2}^{\prime} / A \bar{a} \bar{d}_{1}\right)$. But then $\bar{d}_{2}^{\prime} \in \operatorname{cl}\left(A \bar{d}_{1}\right) \subseteq M$, so we conclude by setting $\bar{d}^{\prime}=\bar{d}_{1} \bar{d}_{2}^{\prime}$. 
We remark that the conclusion of Proposition 5.2, or that of Proposition 4.2, could replace $\aleph_{0}$-homogeneity over models in the definition of a quasiminimal pregeometry structure.

Corollary 5.3. Let $\mathfrak{M}$ be an $L$-structure for a countable language $L$, equipped with a pregeometry cl. Suppose that $\mathfrak{M}$ satisfies (QM1)-(QM4) and is $\aleph_{0}$-homogeneous over $\emptyset$, that is, the conclusion of Lemma 2.2 holds.

Then the following are equivalent.

(a) The structure $\mathfrak{M}$ satisfies (QM5).

(b) If $M \preccurlyeq_{\mathrm{cl}} \mathfrak{M}$ is countable and $\bar{a} \in \mathfrak{M}$, then there is a finite set over which $\operatorname{tp}(\bar{a} / M)$ does not split.

(c) If $M \preccurlyeq$ cl $\mathfrak{M}$ is countable and $\bar{a}, \bar{b} \in \mathfrak{M}$ with $\bar{b} \in \operatorname{cl}(M \bar{a})$, then $\operatorname{tp}(\bar{b} / M \bar{a})$ is s-isolated.

Proof. Proposition 4.2 gives (a) $\Rightarrow$ (b), and the proof of Proposition 5.2 gives (b) $\Rightarrow$ (c).

We show (c) $\Rightarrow$ (a). Let $H, \bar{b}, H^{\prime}, \bar{b}^{\prime}, a$ be as in (QM5), with $H$ and $H^{\prime}$ closed in $\mathfrak{M}$ of dimension at most $\aleph_{0}$. Write $\sigma: H \bar{b} \rightarrow H^{\prime} \bar{b}^{\prime}$ for the given isomorphism. By (c) applied to $H \preccurlyeq$ cl $\mathfrak{M}$, there exists $\bar{c} \in H$ such that $\operatorname{tp}(a / H \bar{b})$ is isolated by $\operatorname{tp}(a / \bar{c} \bar{b})$; let $\bar{c}^{\prime}:=\sigma(\bar{c})$. By $\aleph_{0}$-homogeneity over $\emptyset$, there exists $a^{\prime}$ such that $\operatorname{tp}(\bar{c} \bar{b} a)=\operatorname{tp}\left(\bar{c}^{\prime} \bar{b}^{\prime} a^{\prime}\right)$. Now suppose $\bar{d} \in H$, and let $\bar{d}^{\prime}:=\sigma(\bar{d})$. By $\aleph_{0}$-homogeneity over $\emptyset$, there exists $a^{\prime \prime}$ such that $\operatorname{tp}\left(\bar{d}^{\prime} \bar{c}^{\prime} \bar{b}^{\prime} a^{\prime}\right)=\operatorname{tp}\left(\bar{d} \bar{c} \bar{b} a^{\prime \prime}\right)$, and by the isolation $\operatorname{tp}\left(\bar{d} \bar{c} \bar{b} a^{\prime \prime}\right)=\operatorname{tp}(\bar{d} \bar{c} \bar{b} a)$. So $\operatorname{tp}(H \bar{b} a)=\operatorname{tp}\left(H^{\prime} \bar{b}^{\prime} a^{\prime}\right)$ as required.

\section{Excellence}

Shelah's notion of excellence says that types over certain configurations we call crowns are determined over finite sets, that is, s-isolated. It will be convenient to use notation for crowns that is borrowed from the notation used in simplicial complexes, in particular with the use of a boundary operator $\partial$.

Let $M$ be an infinite-dimensional model, let $B \subseteq M$ be an independent subset of cardinality $\aleph_{0}$, write $M_{B}=\operatorname{cl}(B)$, and let $b_{1}, \ldots, b_{n} \in B$ be distinct. We define $\partial_{i} M_{B}=\operatorname{cl}\left(B \backslash\left\{b_{i}\right\}\right)$ and the $n$-crown $\partial M_{B}=\bigcup_{i=1}^{n} \partial_{i} M_{B}$. Note that $\partial M_{B}$ depends on $n$ and the choice of $b_{1}, \ldots, b_{n}$, but we suppress that from the notation.

Definition 6.1. The model $M$ is excellent if for every $n \in \mathbb{N}$ with $n \geqslant 2$ and every $n$-crown $\partial M_{B}$ in $M$, and every finite tuple $\bar{a} \in M_{B}$, then the type $\operatorname{tp}\left(\bar{a} / \partial M_{B}\right)$ is s-isolated.

Note that the definition of crown here, and consequently the definition of excellence, is a special case of the definition in [6]. However, it is exactly the special case that is used in the proofs in that paper.

Proposition 6.2. For each $n \geqslant 2$, each $n$-crown $\partial M_{B}$ and $\bar{a} \in M_{B}$ we have

(i) $\operatorname{tp}\left(\bar{a} / \partial M_{B}\right)$ is s-isolated and

(ii) if $\operatorname{tp}\left(\bar{c} / \partial M_{B}\right)=\operatorname{tp}\left(\bar{a} / \partial M_{B}\right)$, then there is $\pi \in \operatorname{Aut}\left(M_{B} / \partial M_{B}\right)$ such that $\pi(\bar{a})=\bar{c}$.

In particular, $M$ is excellent.

Proof. Any two $n$-crowns in $M$ are isomorphic, so we may fix $B$ and assume $M=M_{B}$. We proceed by induction on $n$. The proofs for the base case $n=2$ and the inductive step are very similar, so we do them together. Thus, we suppose that the result holds up to $n-1$ for some $n \geqslant 2$. 
Fix $b_{1}, \ldots, b_{n} \in B$, and let $\bar{a} \in M$ be a finite tuple. Choose $b_{0} \in B \backslash\left\{b_{1}, \ldots, b_{n}\right\}$ such that $\bar{a} \in \operatorname{cl}\left(B \backslash\left\{b_{0}\right\}\right)$ and let $M^{\prime}=\operatorname{cl}\left(B \backslash\left\{b_{0}\right\}\right)$. Choose $\pi \in \operatorname{Aut}\left(M / \operatorname{cl}\left(B \backslash\left\{b_{0}, b_{n}\right\}\right)\right)$ such that $\pi\left(b_{n}\right)=b_{0}$ and $\pi\left(b_{0}\right)=b_{n}$.

First, suppose $n=2$. Then $\bar{a}, \pi(\bar{a}) \in \operatorname{cl}\left(\partial_{1} M, b_{1}\right)$ so, by Proposition $5.2, \operatorname{tp}\left(\bar{a}, \pi(\bar{a}) / \partial_{1} M, b_{1}\right)$ is s-isolated.

Now suppose $n>2$. Then $\Lambda:=\bigcup_{i=1}^{n-1} \partial_{i} M$ is an $(n-1)$-crown and $\operatorname{cl}(\Lambda)=M$ so, by part (i) of the induction hypothesis, $\operatorname{tp}(\bar{a}, \pi(\bar{a}) / \Lambda)$ is s-isolated. Note that in this case $b_{1} \in \Lambda$, so $\operatorname{tp}\left(\bar{a}, \pi(\bar{a}) / b_{1}, \Lambda\right)$ is s-isolated. Thus (whatever $n$ is) $\operatorname{tp}\left(\bar{a} / b_{1}, \pi(\bar{a}), \bigcup_{i=1}^{n-1} \partial_{i} M\right)$ is s-isolated, say over $A_{0}$. Since $\pi(\bar{a}) \subseteq \partial_{n} M$, we have $A_{0} \subseteq \partial M$.

We proceed to show that $\operatorname{tp}(\bar{a} / \partial M)$ is s-isolated over $A_{0}$. So suppose $\bar{c} \in M$ and $\operatorname{tp}\left(\bar{c} / A_{0}\right)=\operatorname{tp}\left(\bar{a} / A_{0}\right)$. Then we have $\operatorname{tp}\left(\bar{c} / b_{1}, \pi(\bar{a}), \bigcup_{i=1}^{n-1} \partial_{i} M\right)=\operatorname{tp}\left(\bar{a} / b_{1}, \pi(\bar{a}), \bigcup_{i=1}^{n-1} \partial_{i} M\right)$, so by Lemma 3.1 (if $n=2$ ) or by part (ii) of the inductive hypothesis (if $n>2$ ) there is $\sigma \in \operatorname{Aut}\left(M / b_{1}, \pi(\bar{a}), \bigcup_{i=1}^{n-1} \partial_{i} M\right)$ such that $\sigma(\bar{a})=\bar{c}$. Let $\eta$ be the commutator $\eta=\sigma \pi^{-1} \sigma^{-1} \pi$. Then since $\pi(\bar{a})$ is fixed by $\sigma^{-1}$, we have $\eta(\bar{a})=\sigma(\bar{a})=\bar{c}$. Now $\eta$ fixes $\bigcup_{i=1}^{n} \operatorname{cl}\left(B \backslash\left\{b_{0}, b_{i}\right\}\right)=$ $\partial M^{\prime}$ pointwise; indeed, for $i=1, \ldots, n-1$ we have $\operatorname{cl}\left(B \backslash\left\{b_{0}, b_{i}\right\}\right) \subseteq \operatorname{cl}\left(B \backslash\left\{b_{i}\right\}\right)$, and the latter is fixed setwise by $\pi$ and pointwise by $\sigma$, while $\operatorname{cl}\left(B \backslash\left\{b_{0}, b_{n}\right\}\right)$ is fixed pointwise by $\pi$ and setwise by $\sigma$. So $\operatorname{tp}\left(\bar{c} / \partial M^{\prime}\right)=\operatorname{tp}\left(\bar{a} / \partial M^{\prime}\right)$.

Let $B_{0}$ be a finite subset of $B \backslash\left\{b_{0}, b_{1}, \ldots, b_{n}\right\}$ such that $\bar{a} \in \operatorname{cl}\left(B_{0} \cup\left\{b_{1}, \ldots, b_{n}\right\}\right)$. Then also $\bar{c} \in \operatorname{cl}\left(B_{0} \cup\left\{b_{1}, \ldots, b_{n}\right\}\right)$, since $B_{0} \cup\left\{b_{1}, \ldots, b_{n}\right\} \subseteq \bigcup_{i=1}^{n-1} \partial_{i} M \cup\left\{b_{1}\right\}$.

Since $B$ is infinite, there is a bijection $B \backslash\left(B_{0} \cup\left\{b_{0}, b_{1}, \ldots, b_{n}\right\}\right) \rightarrow B \backslash\left(B_{0} \cup\left\{b_{1}, \ldots, b_{n}\right\}\right)$ extending to an isomorphism $\phi: M^{\prime} \rightarrow M$ that fixes $\operatorname{cl}\left(B_{0} \cup\left\{b_{1}, \ldots, b_{n}\right\}\right)$ pointwise.

Now $\operatorname{tp}\left(\bar{c} / \partial M^{\prime}\right)=\operatorname{tp}\left(\bar{a} / \partial M^{\prime}\right)$, so $\operatorname{tp}\left(\phi(\bar{c}) / \phi\left(\partial M^{\prime}\right)\right)=\operatorname{tp}\left(\phi(\bar{a}) / \phi\left(\partial M^{\prime}\right)\right) ;$ but $\phi(\bar{a})=\bar{a}$ and $\phi(\bar{c})=\bar{c}$, and $\phi\left(\partial M^{\prime}\right)=\partial M$, so $\operatorname{tp}(\bar{c} / \partial M)=\operatorname{tp}(\bar{a} / \partial M)$. Thus, part (i) is proved.

For (ii), suppose that $\operatorname{tp}(\bar{c} / \partial M)=\operatorname{tp}(\bar{a} / \partial M)$, and so in particular $\operatorname{tp}\left(\bar{c} / A_{0}\right)=\operatorname{tp}\left(\bar{a} / A_{0}\right)$. Let $\eta \in \operatorname{Aut}\left(M / \partial M^{\prime}\right)$ and $\phi: M^{\prime} \rightarrow M$ be as above. Since $\eta$ fixes $B$ pointwise, $\eta \mid M^{\prime} \in$ $\operatorname{Aut}\left(M^{\prime} / \partial M^{\prime}\right)$. Defining $\theta=\phi \circ\left(\eta \mid M^{\prime}\right) \circ \phi^{-1}$, we have $\theta \in \operatorname{Aut}(M / \partial M)$ with $\theta(\bar{a})=\bar{c}$, which proves (ii).

\section{Quasiminimal structures}

An uncountable structure $M$ is quasiminimal if every first-order $M$-definable subset of $M$ is countable or co-countable. In this section, we treat the question of when a quasiminimal structure is a quasiminimal pregeometry structure. Certainly some conditions are required, for example, $\omega_{1} \times \mathbb{Q}$ equipped with the lexicographic order has quantifier elimination and is quasiminimal, but the quasiminimal closure $\left(\mathrm{cl}_{p}\right.$ defined below) does not satisfy exchange.

Based on the analyses of Zilber [10] and Pillay-Tanović [7], we are able to give simple 'natural' criteria that, under the assumption of quasiminimality, substitute for all the conditions of quasiminimal pregeometry structures other than (QM5). For (QM5), we have no alternative formulation in this context beyond those given in Corollary 5.3.

So let $M$ be an uncountable quasiminimal structure in a countable language. Suppose, extending the language if necessary, quantifier elimination for types realized in $M$ : if $\bar{a} \in M$ and $\bar{b} \in M$ have the same quantifier-free type, then $\bar{a}$ and $\bar{b}$ have the same first-order type.

Let $p \in S_{1}(M)$ be the generic type, the type consisting precisely of the co-countable formulas. For $A \subseteq M$, define $\operatorname{cl}_{p}(A):=\left\{x \in M \mid x \not \models p_{A}\right\}$. A weak Morley sequence in $p$ over $A \subseteq M$ is a sequence $\left(a_{1}, \ldots\right)$ such that $a_{i} \in M$ and $\operatorname{tp}\left(a_{i} / A a_{<i}\right)=p_{A a_{<i}}$, where $a_{<i}:=\left\{a_{j} \mid j<i\right\}$.

Proposition 7.1. (A) The structure $\left(M, \mathrm{cl}_{p}\right)$ is a quasiminimal pregeometry structure if

(i) $p$ does not split over $\emptyset$; that is, if $\operatorname{tp}(\bar{b} / \emptyset)=\operatorname{tp}\left(\bar{b}^{\prime} / \emptyset\right)$, then for all $\phi$ we have $\phi(x, \bar{b}) \in p$ if and only if $\phi\left(x, \bar{b}^{\prime}\right) \in p$, that is, $|\phi(M, \bar{b})|=\left|\phi\left(M, \bar{b}^{\prime}\right)\right|$; 
(ii) there is no $M$-definable partial order on $M$, defined over finite $A \subseteq M$ say, for which weak Morley sequences in $p$ over $A$ are increasing;

(iii) $\left(M, \mathrm{cl}_{p}\right)$ satisfies (QM5).

(B) Conversely, if $(M, \mathrm{cl})$ is an uncountable quasiminimal pregeometry structure, then $M$ is a quasiminimal structure, $\mathrm{cl}=\mathrm{cl}_{p}$, and (i)-(iii) hold.

Proof. (A) By Pillay and Tanović [7, Theorem 4], (i) and (ii) imply that $\mathrm{cl}_{p}$ is indeed a pregeometry. Axioms (QM1-3) are clear. Axiom (QM4) follows directly from (i).

(B) By (QM1) and (QM4), there exists a complete type $q \in S_{1}(M)$ such that for $B \subseteq M$ finite and $a \in M$, we have $a=q_{B}$ if and only if $a \notin \operatorname{cl}(B)$. By (QM3) and uncountability of $M$, a formula $\phi(x, \bar{b}) \notin q$ if and only if $\phi(M, \bar{b})$ is countable. So $M$ is quasiminimal, $q=p$, and hence $\mathrm{cl}=\mathrm{cl}_{p}$.

To prove (i), suppose $\phi(M, \bar{b}) \in p$ and $\operatorname{tp}(\bar{b} / \emptyset)=\operatorname{tp}\left(\bar{b}^{\prime} / \emptyset\right)$. Then say $a \in \phi(M, \bar{b}) \backslash \operatorname{cl}(\bar{b})$. By (QM5), there exists $a^{\prime} \in M$ such that $\operatorname{tp}(a, \bar{b})=\operatorname{tp}\left(a^{\prime}, \bar{b}^{\prime}\right)$. By (QM1), $a^{\prime} \notin \operatorname{cl}\left(\bar{b}^{\prime}\right)$. Hence, $\phi\left(M, \bar{b}^{\prime}\right) \in p$.

To prove (ii), note that permutations of weak Morley sequences are weak Morley sequences, since $\mathrm{cl}_{p}=\mathrm{cl}$ is a pregeometry.

REMARK 7.2. Conditions (i) and (ii) in the preceding proposition could be replaced with the following conditions of a more elementary flavour, which appear in [10]:

(i') $\aleph_{0}$-homogeneity over $\emptyset$;

(ii') 'Countability is weakly definable in $M^{\prime}$ ': if $a \in \mathrm{cl}_{p}(\bar{b})$, then there exists a formula $\phi(x, \bar{y})$ over $\emptyset$ such that $M \models \phi(a, \bar{b})$ and $\left|\phi\left(M, \bar{b}^{\prime}\right)\right| \leqslant \aleph_{0}$ for all $\bar{b}^{\prime} \in M$;

(iii') $|M|>\aleph_{1}$ or there is no definable partial order on $M$ with a chain in $M$ of order type $\omega_{1}$

Indeed, (i') and (ii') imply (i), since if $\psi(M, \bar{b})$ is countable, it is then covered by countably many $\phi_{i}(M, \bar{b})$ as in (ii'); by (i'), if $\operatorname{tp}\left(\bar{b}^{\prime}\right)=\operatorname{tp}(\bar{b})$, then $\psi\left(M, \bar{b}^{\prime}\right)$ is countable since it is covered by the countably many countable $\phi_{i}\left(M, \bar{b}^{\prime}\right)$. By Zilber [10, Lemma 3.0.3], (ii') and (iii') imply that $\mathrm{cl}_{p}$ is a pregeometry and hence that weak Morley sequences in $p$ are indiscernible, and hence that (ii) holds by Pillay and Tanović [7, Theorem 4]; conversely, [7, Theorem 4] shows under the assumption of (i) and (ii) that $p$ is $\emptyset$-definable, which implies (ii'), and that $\mathrm{cl}_{p}$ satisfies exchange, which implies (iii').

Acknowledgement. The authors would like to thank the Max Planck Institute for Mathematics, Bonn, where some of this work was done.

\section{References}

1. J. T. Baldwin, Categoricity, University Lecture Series 50 (American Mathematical Society, Providence, RI, 2009).

2. M. BAys and J. Kirby, 'Excellence and uncountable categoricity for Zilber's exponential fields', Preprint, 2013, arXiv:1305.0493 [math.LO].

3. M. BAYs and B. ZILBER, 'Covers of multiplicative groups of algebraically closed fields of arbitrary characteristic', Bull. London Math. Soc. 43 (2011) 689-702.

4. B. HART, 'An exposition of OTOP', Classification theory (Chicago, IL, 1985), Lecture Notes in Mathematics 1292 (Springer, Berlin, 1987) 107-126.

5. T. Hyttinen and M. KesëLё, 'Independence in finitary abstract elementary classes', Ann. Pure Appl. Logic 143 (2006) 103-138.

6. J. Kirby, 'On quasiminimal excellent classes', J. Symbolic Logic 75 (2010) 551-564. 
7. A. Pillay and P. Tanović, 'Generic stability, regularity, and quasiminimality', Models, logics, and higher-dimensional categories, CRM Proceedings \& Lecture Notes 53 (American Mathematical Society, Providence, RI, 2011) 189-211.

8. S. Shelah, 'Classification theory for nonelementary classes. I. The number of uncountable models of $\psi \in L_{\omega_{1}, \omega}$. Part A', Israel J. Math. 46 (1983) 212-240.

9. S. Shelah, Classification theory and the number of nonisomorphic models, 2nd edn, Studies in Logic and the Foundations of Mathematics 92 (North-Holland Publishing, Amsterdam, 1990).

10. B. Zilber, Elements of geometric stability theory, Lecture Notes (2003), http://people.maths.ox.ac. uk/ zilber/est.pdf

11. B. Zilber, 'Pseudo-exponentiation on algebraically closed fields of characteristic zero', Ann. Pure Appl. Logic 132 (2005) 67-95.

12. B. Zilber, 'A categoricity theorem for quasi-minimal excellent classes', Logic and its applications, Contemporary Mathematics 380 (American Mathematical Society, Providence, RI, 2005) 297-306.

13. B. ZILBER, 'Covers of the multiplicative group of an algebraically closed field of characteristic zero', J. London Math. Soc. (2) 74 (2006) 41-58.

Martin Bays and Bradd Hart

Department of Mathematics and Statistics McMaster University

1280 Main St. W

Hamilton

Ontario

Canada $L 8 S 4 L 8$

mbays@sdf.org

hartb@math.mcmaster.ca

Jonathan Kirby

School of Mathematics

University of East Anglia

Norwich Research Park

Norwich

NR4 7TJ

United Kingdom

jonathan.kirby@uea.ac.uk
Tapani Hyttinen and Meeri Kesälä

Department of Mathematics and Statistics

University of Helsinki

PO Box 68 (Gustaf Hällströmin katu 2b)

FI-00014 Helsinki

Finland

tapani.hyttinen@helsinki.fi

meeri.kesala@helsinki.fi 